\title{
Impacto del gasto público en la calidad del sistema de salud de México
}

\section{Impact of public spending on the quality of the health system of Mexico}

\author{
ZÁRATE-MARTÍNEZ, María Guadalupe †*, AGUILERA-ARREDONDO, Paulina y JIMÉNEZ-RICO, \\ Artemio
}

Universidad de Guanajuato, División de Ciencias Económico-Administrativas, Guanajuato, Guanajuato, México.

ID $1^{\text {er }}$ Autor: María Guadalupe, Zárate-Martínez / ORC ID: 0000-0001-5834-1113, Researcher ID Thomson: AAG-37662020, CVU CONACYT ID: 1047513

ID $1^{\text {er }}$ Coautor: Paulina, Aguilera-Arredondo / ORC ID: 0000-0002-5355-0749, Researcher ID Thomson: AAG-37682020, CVU CONACYT ID: 1047503

ID $2^{\text {do }}$ Coautor: Artemio, Jiménez-Rico / ORC ID: 0000-0001-9069-6483, Researcher ID Thomson: S-7880-2018, CVU CONACYT ID: 947479

DOI: $10.35429 / J E D \cdot 2020.22 .7 .1 .10$

Recibido: Enero 10, 2020; Aceptado: Marzo 30, 2020

\begin{abstract}
Resumen
La salud se considera un Derecho Humano que toda persona debe recibir de manera gratuita y de calidad, su importancia radica en que es un servicio básico para el desarrollo de un país. En México el Gasto Público en salud equivale al $2.5 \%$ del PIB, sin embargo, la OCDE indica que se requiere destinar por lo menos un $6 \%$ para tener un Sistema de Salud óptimo. En ese mismo tenor, México cuenta con 2.9 enfermeras por cada mil habitantes, mientras que Noruega cuenta con 17.8 enfermeras por cada mil. El objetivo de esta investigación es analizar el gasto público destinado al sector salud para determinar su impacto en los principales indicadores que miden la calidad del servicio brindado por el Sistema de Salud Mexicano. Algunos resultados indican que México enfrenta necesidades de salud complejas y retadoras y que existen enormes discrepancias en comparación con otros países. Esta investigación contribuye en proporcionar una perspectiva más amplia sobre el Gasto Público asignado al Sistema de Salud de México con la finalidad de diseñar políticas públicas que permitan ofrecer una atención de alta calidad centrada en las personas.
\end{abstract}

Gasto público, Sistema de Salud, Calidad

\begin{abstract}
Health is considered a Human Right that every person should receive for free and in quality, it is important in the fact that it is a basic service for the development of a country. In Mexico, public spending on health is equivalent to $2.5 \%$ of GDP, however, the OECD indicates that it is necessary to allocate at least $6 \%$ to have an optimal Health System. In the same tenor, Mexico has 2.9 nurses per thousand inhabitants, while Norway has 17.8 nurses per thousand. The objective of this research is to analyze public spending for the health sector to determine its impact on the main indicators that measure the quality of service provided by the Mexican Health System. Some results indicate that Mexico faces complex and challenging health needs and that there are huge discrepancies compared to other countries. This research contributes to providing a broader perspective on the public expenditure allocated to the Health System of Mexico in order to design public policies that allow offering high-quality care focused on people.
\end{abstract}

Public expenditure, Health System, Quality

Citación: ZÁRATE-MARTÍNEZ, María Guadalupe, AGUILERA-ARREDONDO, Paulina y JIMÉNEZ-RICO, Artemio. Impacto del gasto público en la calidad del sistema de salud de México. Revista de Desarrollo Económico. 2020. 7-22:1-10.

\footnotetext{
* Correspondencia al Autor: (Correo electrónico: mg.zaratemartinez@ugto.mx)

$\dagger$ Investigador contribuido como primer autor.
} 


\section{Introducción}

De acuerdo con la Organización Mundial de la Salud (OMS), "la salud es un estado de completo bienestar físico, mental y social, y no solamente la ausencia de afecciones o enfermedades" (OMS, 1948, p.1).

El derecho a la salud pertenece a uno de los Derechos Humanos que toda persona debe poseer por el sólo hecho de serlo. El Estado debe brindar el acceso a la salud de manera gratuita y de calidad ya que es, además, un servicio básico para el desarrollo de un país.

La OMS, destaca que un Sistema de Salud es "la suma de las organizaciones, instituciones y recursos cuyo objetivo principal consiste en mejorar la salud. Tiene que proporcionar buenos tratamientos y servicios que respondan a las necesidades de la población y sean justos desde el punto de vista financiero" (OMS, 2005, p.1).

Partiendo de la anterior premisa, el
Sistema de Salud en México
desafortunadamente no cumple cabalmente, ya
que enfrenta necesidades de salud complejas y
retadoras, además de que las personas no pueden
elegir a su proveedor de servicios, debido a que
éstos los determina su empleo.

La Organización para la Cooperación y Desarrollo Económicos (OCDE), recomienda que por cada 1,000 habitantes existan 3.5 doctores, mientras que en México se tienen 2.4 doctores posicionándose en los últimos lugares respecto a otros países miembros de la OCDE (OCDE, 2018).

Asimismo, el Centro de Investigación Económica y Presupuestaria (CIEP) destaca que el Gasto Público en Salud para 2019 equivale en México a apenas el $2.5 \%$ del PIB, muy por debajo del $6 \%$ que estima la OCDE que se necesita para proveer un Sistema de Salud óptimo (CIEP, 2019).

Actualmente el Sistema de Salud Mexicano se enfrenta a las posibles deficiencias hacia la atención brindada a los derechohabientes, motivo por el cual, surge el cuestionamiento sobre si la calidad del servicio está relacionada con los recursos financieros que se les ortorga a las instituciones.
Por lo antes expuesto, el objetivo de esta investigación es analizar la relación existente entre la calidad del servicio que brinda el Sistema de Salud Mexicano y el Gasto Público destinado a este sector para la prestación de dicho servicio. En este sentido, se analizaron indicadores que miden la calidad del servicio de salud y la tendencia del Gasto Público de los últimos años.

Este estudio contribuye a proporcionar una perspectiva sobre la aplicación del Gasto Público en el Sistema de Salud y la situación que posee México en relación con otros países de acuerdo con los estándares de la OCDE, con el fin de diseñar políticas públicas que permitan brindar un servicio de salud de calidad.

\section{Revisión de la literatura}

\section{Gasto Público en el sistema de salud de México}

El Gasto Público es referido como el conjunto de erogaciones que efectúan los Poderes de la Unión y las entidades gubernamentales (el Ejecutivo, considerando el Gobierno Federal, los estatales y municipales y los poderes Legislativos y Judicial, así como los órganos autónomos y el sector paraestatal) en el ejercicio de sus funciones. La acepción más generalizada es la que hace referencia al gasto del Sector Público Federal (Gutiérrez, 2015).

Por otro lado, Vera (2009) menciona que el Gasto Público son todas las erogaciones que realiza la entidad estatal con el propósito de beneficiar a sus habitantes, para que éstos puedan adquirir beneficios con los programas y proyectos que el Estado les ofrece y de esta manera poder satisfacer sus necesidades fundamentales permitiéndoles una mejor inclusión social en el entorno.

Ahora bien, el Sistema de Salud Mexicano nace en 1943, siendo en la actualidad una amalgama de instituciones públicas y privadas creadas para el acceso a la salud y servicios de calidad para todos. El Sistema Mexicano de salud comprende dos sectores, el público y el privado. Dentro del sector público, se encuentran las instituciones que se muestran en la figura 1. 
Mientras que, el sector privado comprende a las compañías aseguradoras y los prestadores de servicios que trabajan en consultorios, clínicas y hospitales privados, incluyendo a los prestadores de servicios de medicina alternativa (Corona, 2017).

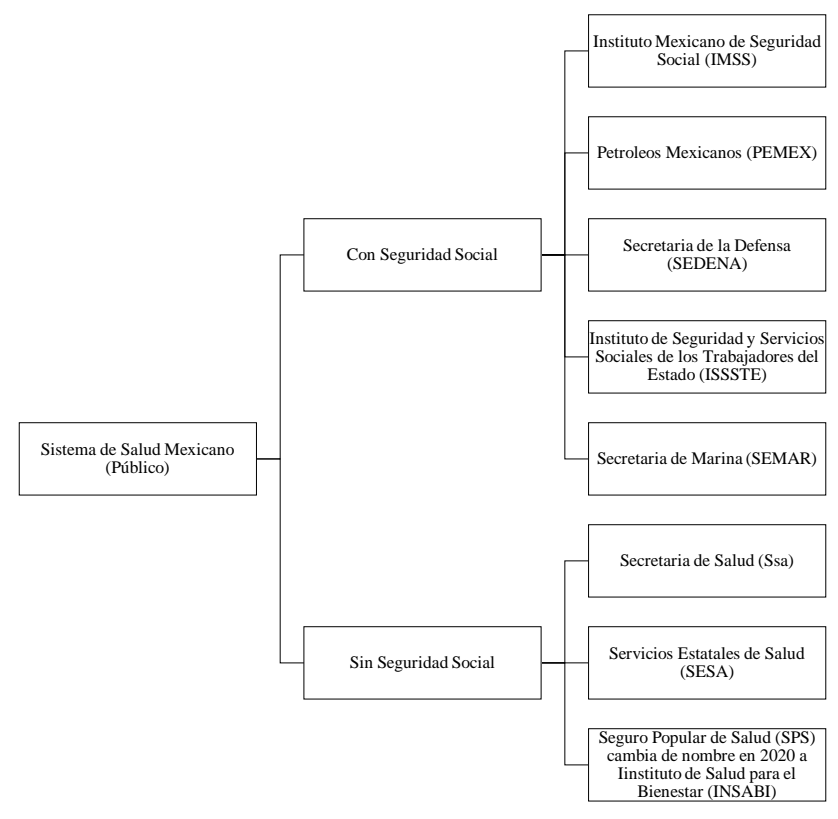

Figura 1 Instituciones de Salud en México Fuente: elaboración propia [Word] con datos de Corona (2017)

Gómez et al. (2011) exponen que el Sistema Mexicano de salud ofrece beneficios en salud muy diferentes dependiendo de la población de que se trate. En México hay tres distintos grupos de beneficiarios de las instituciones de salud:

- $\quad$ Los trabajadores asalariados, los jubilados y sus familias.

- Los auto empleados, trabajadores del sector informal, desempleados y personas que se encuentran fuera del mercado de trabajo y sus familias.

- La población con capacidad de pago (es decir quienes acuden al sector privado).

\section{El Instituto Mexicano de Seguridad} Social (IMSS) cuenta con un régimen obligatorio, que protege a la mayoría de sus afiliados, y un régimen voluntario. Los afiliados al Instituto de Seguridad y Servicios Sociales de los Trabajadores del Estado (ISSSTE), que son todos empleados del Gobierno del Estado y sus familias, los pensionados y jubilados, cuentan con un conjunto de beneficios similares a los que ofrece el IMSS.
Los asegurados de Petróleos Mexicanos (PEMEX), Secretaría de la Defensa Nacional (SEDENA) y Secretaría de la Marina (SEMAR) cuentan con beneficios parecidos a los del IMSS e ISSSTE. El Seguro Popular de Salud (SPS) garantiza el acceso personas sin seguridad social a un paquete con alrededor de 260 intervenciones de salud, con sus respectivos medicamentos, también ofrece un paquete de 18 intervenciones de alto costo. La población no asegurada recibe también en las unidades de los Servicios Estatales de Salud, beneficios muy heterogéneos: servicios ambulatorios básicos en los centros de salud rurales y un conjunto más o menos amplio de intervenciones en las grandes ciudades (Gómez et al., 2011).

Para el año 2020, el SPS cambia de nombre a Instituto de Salud para el Bienestar (INSABI) por decreto del presidente de México Andrés Manuel López Obrador, de acuerdo con Miranda (2020), el INSABI brindará servicios de salud y medicamentos gratuitos en primer y segundo nivel para quienes no cuenten con seguridad social.

Los servicios de salud que prestan las instituciones de seguridad social a sus afiliados se financian con contribuciones del empleador (que en el caso del ISSSTE, PEMEX y Fuerzas Armadas es el gobierno), contribuciones del empleado y contribuciones del gobierno. La Secretaría de Salud (SSA) y los Servicios Estatales de Salud (SESA) se financian con recursos del Gobierno Federal (en su mayoría), con gobiernos estatales y los pagos que los usuarios hacen al momento de recibir la atención (Gómez et al., 2011).

Cabe aclarar que de acuerdo con Miranda (2020), el ISABI ofrece los servicios de primer y segundo nivel de forma gratuita, mientras que el tercer nivel mantiene una cuota de recuperación; entendiendo primer nivel como prevensión de salud (ejemplo campañas de vacunación), segundo nivel consultas médicas y tercer nivel intervenciones quirúrgicas, solo por mencionar algunos ejemplos.

Teniendo en cuenta cómo se financian las instituciones pertenecientes al Sistema de Salud Mexicano, se puede observar cómo es el comportamiento del Gasto en materia de salud de estas instituciones. El CIEP (2018) menciona que, en 2016, el Gasto Público fue equivalente a un $3 \%$ del Producto Interno Bruto (PIB).

ZÁRATE-MARTÍNEZ, María Guadalupe, AGUILERAARREDONDO, Paulina y JIMÉNEZ-RICO, Artemio. Impacto del gasto público en la calidad del sistema de salud de México. Revista de Desarrollo Económico. 2020 
Ahora bien, según el CIEP (2019) el Gasto Público en salud en México para 2019 equivale pobremente al $2.5 \%$ del PIB, muy por debajo del $6 \%$ que estima la OCDE que se necesita para proveer un sistema de salud óptimo. En la última década el punto máximo de presupuesto para salud como porcentaje del PIB fue en 2012 con $2.88 \%$ a partir de entonces ha caído hasta ubicarse en $2.50 \%$ del PIB de acuerdo con el Presupuesto de Egresos de la Federación 2020.

\section{Calidad en el servicio de salud}

De acuerdo con el artículo 4 en su última reforma de la Constitución Política de los Estados Unidos Mexicanos en su párrafo cuarto estipula que "Toda persona tiene derecho a la protección de la salud. La Ley definirá las bases y modalidades para el acceso a los servicios de salud y establecerá la concurrencia de la Federación y las entidades federativas en materia de salubridad general, conforme a lo que dispone la fracción XVI del artículo 73 de esta Constitución" (Cámara de Diputados del H. Congreso de la Unión, 2020).

La calidad es un derecho que debe ser garantizado. Por lo tanto, la garantía de la calidad es imperativa en los sistemas de salud y, de manera concreta, en las organizaciones que los conforman (Coronado et al., 2013).

Sin embargo, para la Organización Panamericana de la Salud (OPS) la deficiencia en la calidad de la atención de la salud se puede manifestar de múltiples maneras: acceso limitado a servicios de salud, servicios inefectivos e ineficientes, quejas médicas, costos elevados, insatisfacción de los usuarios y de los profesionales de la salud, afectación de la credibilidad de las instituciones prestadoras y del sector salud en su conjunto. Peor aún, la deficiencia de la calidad en la prestación de servicios de salud llega a significar la pérdida de vidas humanas (OPS, 2015).

Por otro lado, Aguirre (citado en Martínez et al., 1996) considera necesario tener en cuenta que la calidad de la atención puede enfocarse desde tres ángulos diferentes, conforme a la satisfacción de las expectativas del derechohabiente, de la institución y del trabajador mismo.
$\mathrm{Si}$ bien la calidad es un atributo fundamental de cualquier bien o servicio y se debe fomentar en todas las áreas prioritarias para el desarrollo de los individuos; la salud no debe ser la excepción. Sin embargo, representa un gran reto social a nivel mundial, sobre todo, para aquellos países cuyos niveles de desarrollo no han alcanzado estándares deseables y sostenidos para su progreso sanitario y social (Secretaría de Salud, 2015).

De acuerdo con la publicación del foro más grande de Negocios en México dirigido a los profesionales de la salud (ExpoMED), el Sistema Nacional de Salud de México ha mostrado importantes avances durante los últimos años en la mejora de la calidad en la prestación de servicios de salud. No obstante, el cumplimiento de estándares de calidad aún es heterogéneo entre los distintos prestadores y su eficiencia y resolutividad en algunos casos es reducida, lo que genera insatisfacción de los usuarios (ExpoMED, 2019).

La medición de la calidad y la eficiencia de un servicio de salud es una tarea compleja ya que, además de la complejidad intrínseca que conlleva la medición de conceptos abstractos, no pueden ignorarse la variedad de intereses que pueden influir en una evaluación de ese tipo. En los sistemas públicos y gratuitos el estado financía los servicios de salud y es el mayor interesado en que éstos sean brindados con calidad y eficiencia. Mientras que en la eficiencia se consideran los gastos y costos relacionados con la efectividad alcanzada (Jiménez, 2004).

Para González y Gallardo (2012) la calidad es una combinación de beneficios, riesgos y costos, en donde lo fundamental es ofrecer los mayores beneficios con los menores riesgos posibles y a un costo razonable. La atención médica, para ser de calidad, debe ser segura, eficaz, eficiente, equitativa, oportuna y centrada en el paciente.

Asimismo, Jiménez (2004) nos proporciona qué desde el punto de vista de los gestores o administradores de la atención médica, la calidad con que se brinda un servicio de salud no puede separarse de la eficiencia puesto que, si no se tienen en cuenta el ahorro necesario de los recursos disponibles, el alcance de los servicios será menor que el supuestamente posible. 
Igualmente, los países con ingresos altos y medio altos no sólo destinan mayor porcentaje de recursos, especialmente recursos públicos, sino que hacen más eficiente el uso de los mismos por lo que pueden contar con sistemas de seguridad social de mayor calidad (Salinas et al., 2019). El logro de la política social requiere contar con información de calidad que permita la evaluación continua de las acciones implementadas. Desde 1986 inició el Sistema de Encuestas Nacionales de Salud, que se ha constituido como uno de los principales aportes de los sanitaristas mexicanos, teniendo como producto la Encuesta Nacional de Nutrición y Salud (ENSANUT) que es un instrumento de rendición de cuentas y planeación que permite el análisis de los principales indicadores de impacto, desde un punto de vista imparcial y académico (Secretaría de Salud, 2016).

Ahora bien, para el CIEP (2018), la ENSANUT 2016 revela que el $64.8 \%$ de la población hizo uso de las instituciones públicas $y$ el servicio es calificado entre bueno y muy bueno. Mientras que la Encuesta Nacional de Ingresos y Gastos en los Hogares (ENIGH) en el 2016 permitó estimar los tiempos de traslado y espera en la institución de atención, el cual se puede considerar como un indicador de calidad objetivo, pues es reflejo de la oportunidad de atención y de las barreras organizacionales y económicas que enfrenta la población.

Además, recientemente la OCDE presentó el documento "Health at a Glance 2019", en el que se muestran los últimos datos sobre los indicadores clave en salud, las tendencias y el desempeño de los sistemas sanitarios de los países miembros de la organización, países candidatos y asociados (Código F, 2019).

\section{Metodología a desarrollar}

La metodología utilizada en esta investigación por sus características corresponde a un enfoque del tipo cualitativa con un alcance exploratorio. Además, es documental, en virtud de que los datos se obtuvieron a través de la revisión de diversas páginas oficiales de instituciones como la Organización para la Cooperación y el Desarrollo Económicos (OCDE), Secretaría de Salud, entre otras, tratando de identificar la relación del gasto público en el Sistema de Salud Mexicano con algunos indicadores que miden la calidad del servicio de salud brindado a los derechohabientes.
Posteriormente se procedió a la construcción del concentrado de datos y elaboración de gráficas con el propósito de observar el comportamiento de las variables en cuestión. Finalmente, una vez recabados los datos, el análisis consistió en contrastar la información con la finalidad de buscar mayor objetividad en relación con el objetivo y las premisas planteadas en esta investigación.

\section{Resultados}

\section{Gasto Público en el sistema de salud de México}

Conforme a la información publicada por el Gobierno Mexicano del Gasto Público Total en miles de pesos, en la figura 2 se observa que la tendencia del gasto ha ido en aumento, durante 2013 y 2014 el incremento fue poco significativo, mientras que de 2015 a 2017 el aumento fue exponencialmente mayor, teniendo un total de $\$ 626,181$ millones de pesos en 2019 , esto significa que el gobierno mexicano invirtió gran cantidad de recursos financieros en la salud de sus ciudadanos en comparación a 2013.

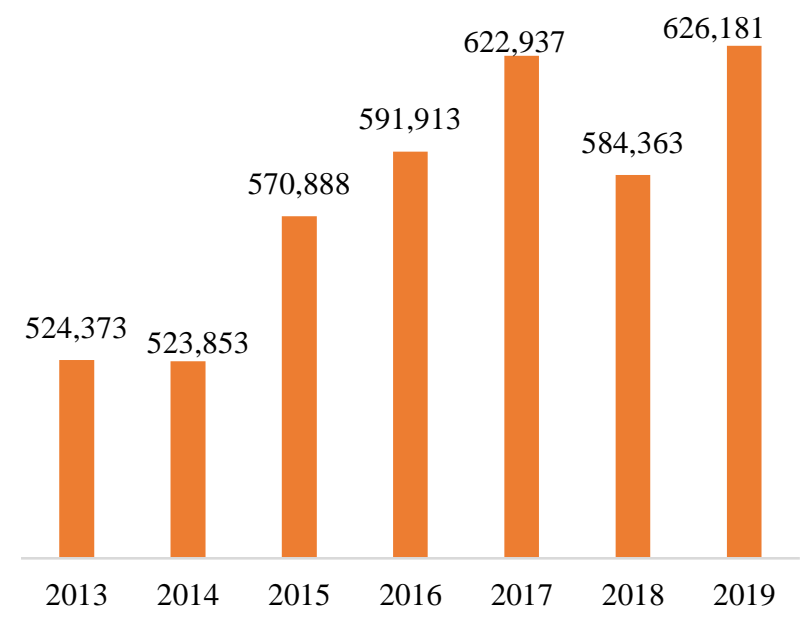

Figura 2 Gasto Público Total en Salud (Millones de Pesos)

Fuente: elaboración propia [Excel] con datos de la Secretaría de Salud (2019) y la Dirección General de Servicios de Documentación Información y Análisis (2019)

De acuerdo con el Ramo 12 de la Cuenta Pública del Gobierno Federal, en el cual se destinan los recursos públicos al sector salud, podemos encontrar que en la figura 3 los programas para la prestación de los servicios de salud se ordenan en cinco subfunciones. Se observa que la subfunción más significativa en el lapso de 2015 a 2019 ha sido la Prestación de Servicios de Salud a la Comunidad. 
De acuerdo con el Senado de la Republica (2018), esta subfunción comprende las acciones de promoción y prevención de salud, atención de urgencias en todos los niveles, entre otras actividades. En 2019 el total destinado a esta subfunción fue de \$397,236 millones de pesos, cabe mencionar que, en 2016, sufrió una disminución como producto de los brotes epidémicos y crisis humanitarias (OMS, 2016). La diferencia entre 2016 y 2019 en este indicador ha sido de $\$ 66,402$ millones de pesos.

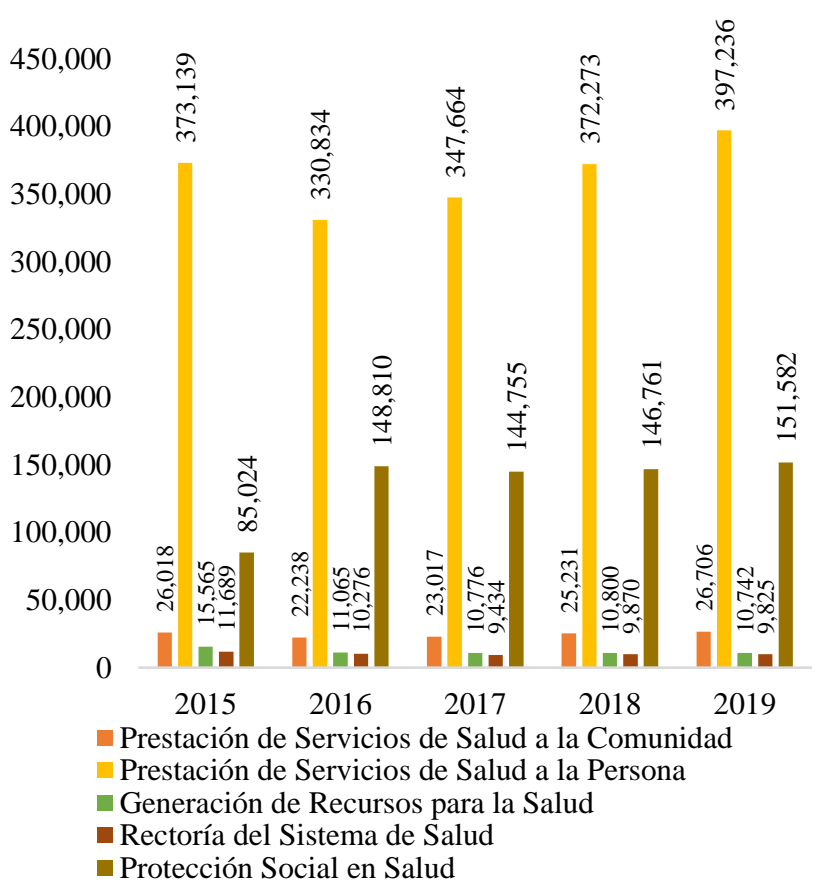

Figura 3 Subfunción del Ramo 12 en millones de pesos Fuente: elaboración propia [Excel] con datos de Código F (2019) basado en el Proyecto de Presupuestos de Egresos de la Federación (PPEF) 2015 a 2019

Por otra parte, los recursos financieros totales para el Ramo 12: Función Salud, de igual manera fue en aumento, teniendo para 2019 un total de $\$ 596,090$ millones de pesos como se observa en la figura 4.

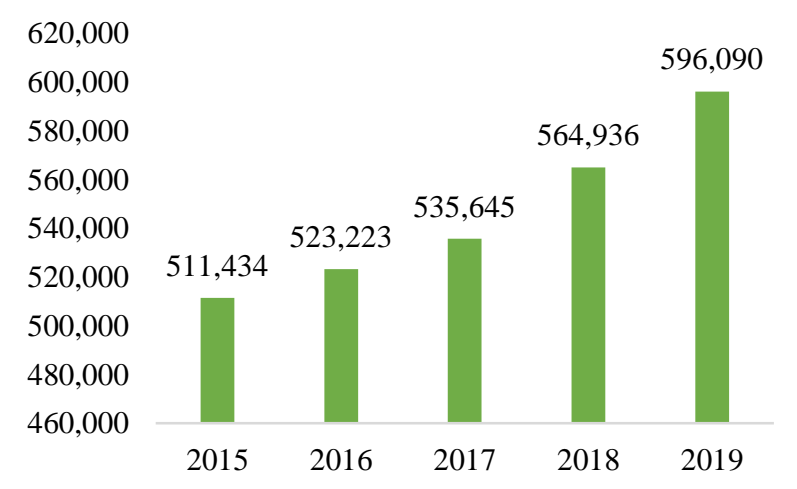

Figura 4 Función Salud en millones de pesos

Fuente: elaboración propia [Excel] con datos de Código F (2019) basado en el PPEF 2015 a 2019
El Ramo 12 muestra también un analítico de las instituciones que brindan el servicio de salud a la población mexicana. En la figura $5 \mathrm{se}$ analiza que la institución que ha recibido una cantidad mayor de recursos financieros del Ramo 12 durante el lapso de los años 2015 2019 es el IMSS. Esta institución cuenta para 2019 con 12 millones 327 mil 845 derechohabientes (IMSS,2019); mientras que la institución con menor monto de recursos públicos asignados es la Secretaría de Marina ya que el número de beneficiarios es menor pues solo brinda el servicio de salud a sus miembros y las familias de éstos.

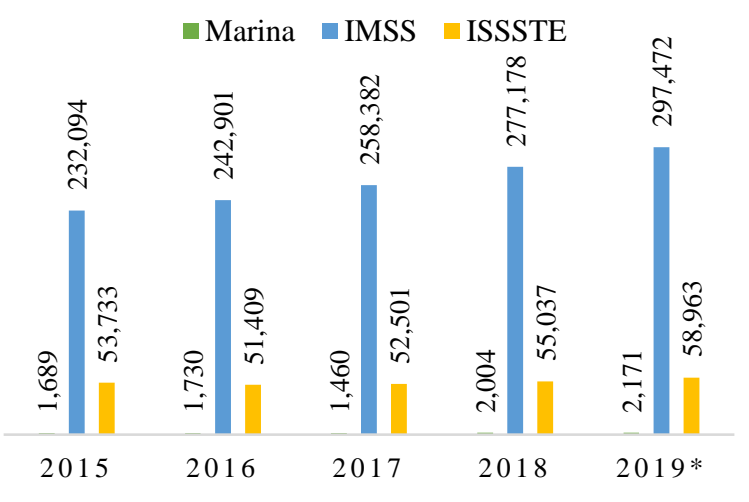

Figura 5 Instituciones de la Función Salud en millones de pesos

Fuente: elaboración propia [Excel] con datos de Código F (2019) basado en el PPEF 2015 a 2019

Pasando al panorama internacional, como se percibe en la figura 6, México se encuentra en el último lugar de los países miembros de la OCDE respecto a la aplicación de recursos públicos para el sector salud per cápita. Lo anterior, se debe porque la OCDE estima que para otorgar un buen servicio de salud se necesitan como mínimo $\$ 3,040.55$ dólares por habitante para 2018, mientras que México sólo destinó $\$ 585.80$ dólares per cápita, es decir sólo el $19.26 \%$ del promedio de la OCDE. Por otro lado, países como Austria destinó \$4,032.59 dólares por habitante para 2018 cantidad por encima del promedio establecido. 




Figura 6 Gasto Público en Salud per cápita en dólares Fuente: elaboración propia [Excel] con datos de Health Expenditure. (OCDE, 2019)

Teniendo en cuenta la posición de México a nivel mundial, se procedió a realizar un análisis de la situación interna del país. En la figura 7 se percibe que, si bien el Gasto Público per cápita para salud en dólares en México ha ido en aumento, tuvo una caída en 2016 que representa el punto más bajo. No obstante, de 2016 a 2018 sólo se han aumentado \$28.03 dólares a la salud de cada habitante en México, un aumento muy pobre para mejorar este servicio esencial.

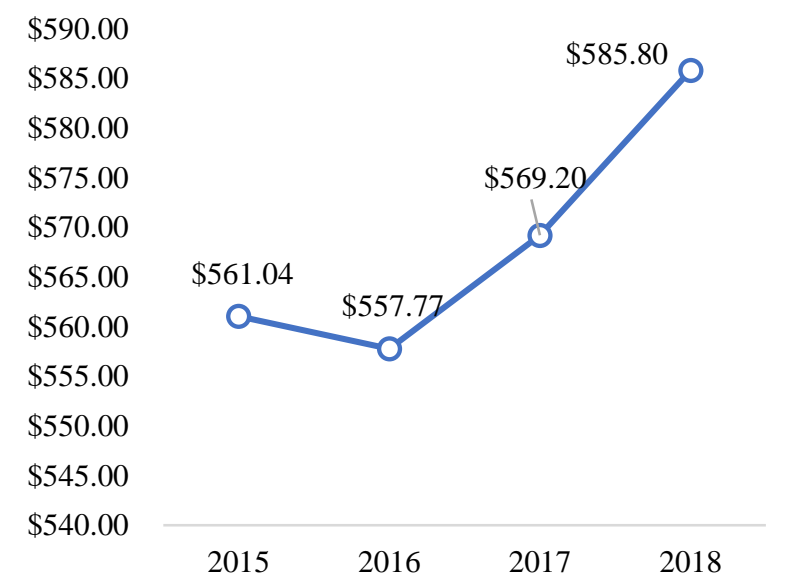

Figura 7 Gasto Público per cápita en México, en dólares Fuente: elaboración propia [Excel] con datos de Health Expenditure (OCDE, 2019)

Ahora bien, en la figura 8 se observa que la OCDE estima que el Gasto Público en salud para brindar una atención de calidad debe abarcar el $6.5 \%$ del Producto Interno Bruto (PIB).
Sin embargo, en 2017 México destinó el $3 \%$ del PIB para la salud, posicionándose en los países más bajos en este indicador, mientras que Alemania asignó un $9.5 \%$ del PIB.

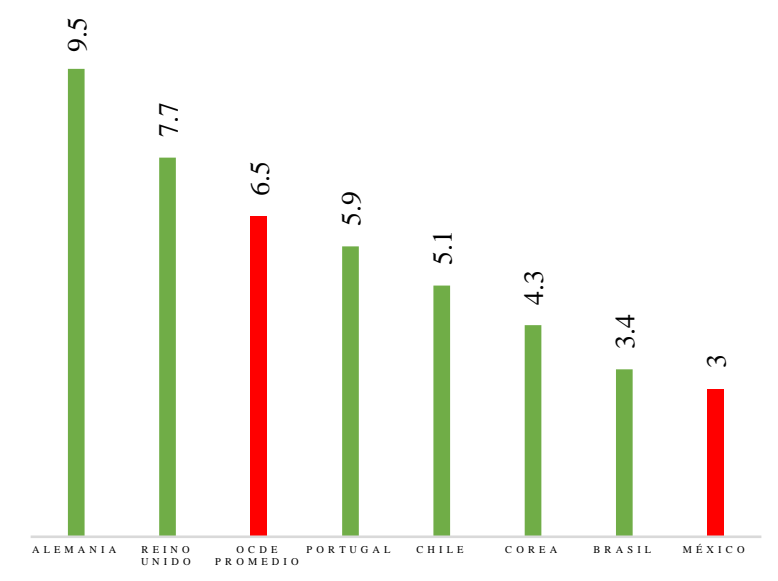

Figura 8 Gasto en Salud como \% del PIB (comparado con países de la OCDE)

Fuente: elaboración propia [Excel] con datos de OCDE (2017)

\section{Calidad en el servicio de salud}

Respecto a la calidad en el servicio de salud, en la figura 9 se analiza el primer indicador, la OCDE establece que el promedio de doctores por cada mil habitantes en 2018 debe ser de 3.5, México está por debajo con 2.43 y Austria es el mejor posicionado en este indicador con 5.18 doctores.

Los médicos se definen como médicos "en ejercicio" que brindan atención directa a los pacientes. Suelen ser generalistas que asumen la responsabilidad de proporcionar atención continua a individuos y familias, o especialistas como pediatras, obstetras / ginecólogos, psiquiatras, especialistas médicos y especialistas quirúrgicos (OCDE, 2018).

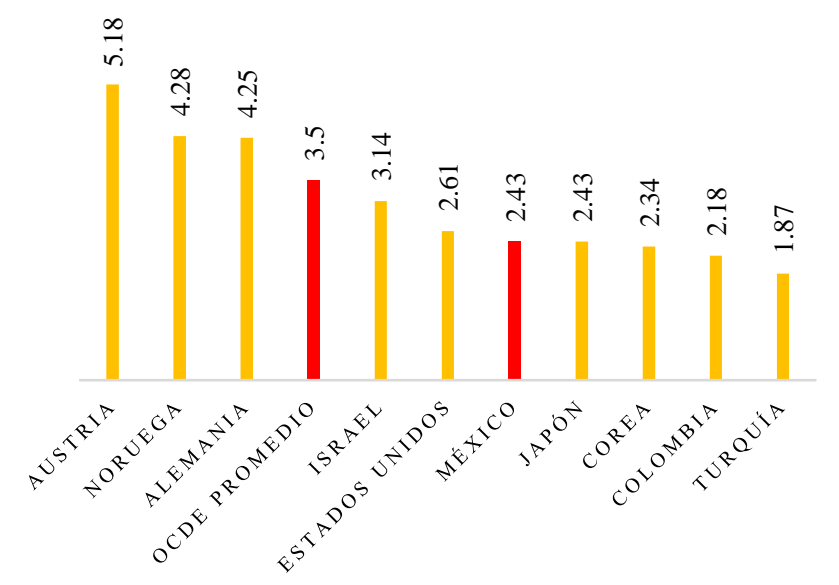

Figura 9 Doctores por cada 1,000 habitantes Fuente: elaboración propia [Excel] con datos de OCDE (2018)

ZÁRATE-MARTÍNEZ, María Guadalupe, AGUILERAARREDONDO, Paulina y JIMÉNEZ-RICO, Artemio. Impacto del gasto público en la calidad del sistema de salud de México. Revista de Desarrollo Económico. 2020 
Las enfermeras se definen como todas las enfermeras "en ejercicio" que prestan servicios de salud directos a los pacientes, incluidas las enfermeras autónomas. Las parteras y auxiliares de enfermería (que no son reconocidas como enfermeras) normalmente están excluidas, aunque algunos países incluyen parteras, ya que se consideran enfermeras especializadas. (OCDE, 2018).

En la figura 10 se muestra el indicador de enfermeras por cada mil habitantes en 218 , el mejor país en este rubro es Noruega, cuenta con 17.8 enfermeras por cada mil, muy por arriba del promedio de la OCDE de 8.8 enfermeras. En contraste, México sólo tiene 2.9 enfermeras por cada mil habitantes, ubicándolo como uno de los peor evaluados en este indicador.

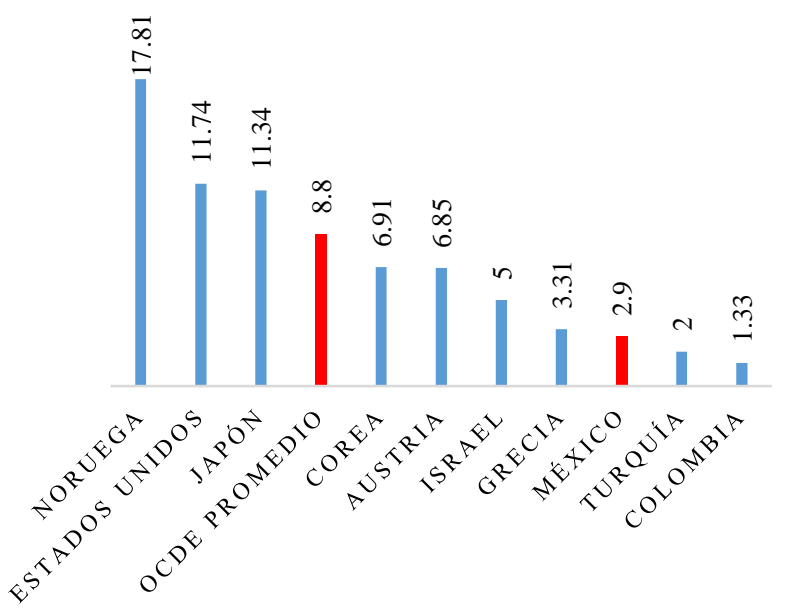

Figura 10 Enfermeras por cada 1,000 habitantes Fuente: elaboración propia [Excel] con datos de OCDE (2018).

El siguiente indicador proporciona una medida de los recursos disponibles para prestar servicios a pacientes hospitalizados en términos de la cantidad de camillas que se mantienen. El total de camillas de hospital incluye camas de atención curativa, camas de atención de rehabilitación, camas de atención a largo plazo y otras camas en hospitales. (OCDE, 2018).

En la figura 11 se observa que en 2018 México tenía 1.38 camillas por cada mil habitantes, resultado muy por debajo del promedio de la OCDE de 4.7 camillas. En comparación, Japón es el país con mayor número de camillas con 13 por cada mil habitantes.

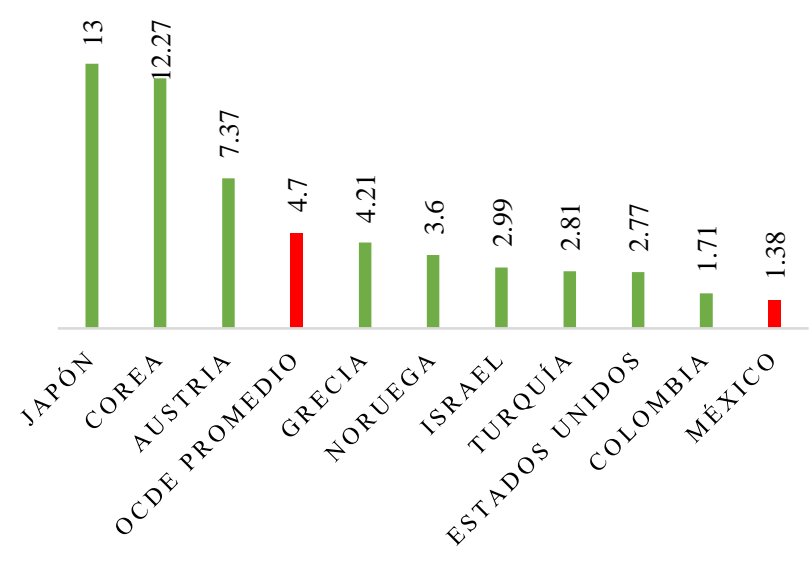

Figura 11 Camillas por cada 1,000 habitantes Fuente: elaboración propia [Excel] con datos de OCDE (2018)

Ahora, de acuerdo con el CIEP, el tiempo adecuado de espera para la atención de salud debe ser de 30 minutos, siendo el IMSS la institución con mayor tiempo de espera para la atención (91 minutos), mientras que en PEMEX el tiempo de espera es de 42 minutos, en el ISSSTE de 55 minutos y en el Seguro Popular de 69 minutos, como se observa en la figura 12.

\section{0 $\begin{array}{lllllll}30 & 42 & 55 & 68 & 69 & 70 & 91\end{array}$ \\ Deseable (referencia) Pemex ISSSTE estatal 0 ISSSTE Seguro Popular IMSS-Prospera IMSS}

Figura 12 Tiempo de espera (minutos) por atención en salud

Fuente: CIEP (2018)

\section{Conclusiones}

El Gasto Público Total en salud en México ha ido en aumento con el paso de los años. Asimismo, el Gasto per cápita en salud incrementó para 2018, aunque no fue un aumento significativo para reducir las grandes brechas en la calidad del servicio de salud respecto a otros páises. En este sentido, México es el país cuyo Gasto per cápita $(\$ 585.80$ dólares, equivalente al $19.26 \%$ del total del promedio de la OCDE \$3,040.55) es el menor de todos los países integrantes de la OCDE. 
La institución a la que se le destina el mayor porcentaje del presupuesto de salud es el IMSS debido a la gran cantidad de derechohabientes con que cuenta. Desafortunadamente, también es la Institución con el mayor tiempo (91 minutos) de espera para la atención de la salud. Respecto al PIB, el porcentaje destinado para Gasto Público en salud en México (2.5\%) se encuentra muy por debajo del promedio de la OCDE (6.3\%) para tener un Sistema de Salud óptimo.

De acuerdo con algunos indicadores básicos para brindar un servicio de salud de calidad establecidos por la OCDE, México se encuentra muy por debajo de los promedios establecidos. Comparando las dos variables de estudio, se concluye que a pesar del aumento en el Gasto Público en salud ha mejorado la calidad en la atención brindada al derechohabiente, sin embargo, no ha sido suficiente para alcanzar los estándares de los indicadores básicos para disminuir las enormes brechas que existen con otros países en el Sistema de Salud.

Por lo tanto, se deben crear políticas públicas o reformar las actuales para mejorar la calidad brindada a los derechohabientes del Sistema de Salud Mexicano.

\section{Referencias}

Cámara de Diputados del H. Congreso de la Unión. (2020). Constitución Política de los Estados Unidos Mexicanos. Última reforma publicada en el Diario Oficial de la Federación el 06 de Marzo de 2020. Recuperado de http://www.diputados.gob.mx/LeyesBiblio/pdf_ mov/Constitucion_Politica.pdf

CIEP. (2018). Sistema Universal de Salud. Retos de Cobertura y Financiamiento. Recuperado de http://saludenmexico.ciep.mx/images/sistemauniversal-de-salud.pdf

CIEP. (2019). La contracción del gasto per cápita en salud: 2010-2020. Recuperado de https://ciep.mx/la-contraccion-del-gasto-percapita-en-salud-2010-2020/

Corona, R. (2017). El Sistema de Salud en México: De la fragmentación hacia un Sistema de Salud Universal. Recuperado de https://www.conaemi.org.mx/singlepost/2017/01/15/El-Sistema-de-Salud-enMéxico-De-la-fragmentación-hacia-unSistema-de-Salud-Universal
Coronado, R., Cruz, E., Macías, S. I., Arellano, A., y Nava, T. I. (2013). El Contexto actual de la calidad en salud y sus indicadores. Revista Mexicana de Medicina Física y Rehabilitación, 25(1). pp. 26-33. Recuperado de https://www.medigraphic.com/pdfs/fisica/mf2013/mf131e.pdf

Código F. (2019). OCDE: Panorama de la Salud 2019. Recuperado de https://codigof.mx/ocdepanorama-de-la-salud-2019/

Código F. (2019). Presupuesto de Salud 2019. Recuperado de https://codigof.mx/presupuestode-salud-2019/

Dirección General de Servicios de Documentación, Información y Análisis. (2019). Cámara de Diputados. Recuperado de http://www.diputados.gob.mx/sedia/sia/se/SAE -ISS-07-19.pdf/

ExpoMED. (2019). La Calidad de la atención a la salud en México a través de sus instituciones. Recuperado de https://expomed.com.mx/lacalidad-de-la-atencion-la-salud-en-mexicotraves-de-sus-instituciones

Gómez, O., Sesma, S., Becerril M., V., Knaul M., F., Arreola, H., y Frenk, J. (2011). Sistema de salud de México. Salud Pública de México, (53), pp. 224-225. Recuperado de http://saludpublica.mx/index.php/spm/article/vi ew/5043/10023

González, L. E., y Gallardo , E. G. (2012). Calidad de la atención médica: La diferencia entre la vida o la muerte. Revista Digital Universitaria (UNAM), 13(8), pp.3. Recuperado de

http://www.revista.unam.mx/vol.13/num8/art81 /art81.pdf

Gutiérrez Lara, A. (2015). Gasto público y Presupuesto Base Cero en México. El Cotidiano, (192), pp. 13-32. Recuperado de https://www.redalyc.org/pdf/325/32539883003. pdf

IMSS. (2019). Estadísticas e Informes. Recuperado de http://www.imss.gob.mx/sites/all/statics/imssBi enestar/estadisticas/01-PoblacionAtendida2019.pdf 
Jiménez Paneque, R. E. (2004). Indicadores de calidad y eficiencia de los servicios hospitalarios. Una mirada actual. Revista Cubana de Salud Pública, 30(1). Recuperado de http://scielo.sld.cu/scielo.php?script=sci_arttext \&pid=S0864-34662004000100004

Martínez, A., Van-Dick, M. Á., Nápoles, F., Robles, J., Ramos, A., y Villaseñor, I. (1996). Hacia una estrategia de garantía de calidad: satisfacción en la utilización de los servicios médicos. Cadernos de Saúde Pública, 12(3), pp.399-403. Recuperado de http://www.scielo.br/scielo.php?script=sci_artte xt\&pid=S0102-311X1996000300013

Miranda, P. (2020). ¿Qué es el INSABI y cómo se puede acceder a los servicios de salud? Recuperado de https://www.eluniversal.com.mx/nacion/comoacceder-los-servicios-de-salud-del-insabi

OCDE. (2017). Panorama de la Salud 2017. Recuperado de https://www.oecdilibrary.org/docserver/9789264306035es.pdf?expires $=1585853603 \& i d=i d \&$ accname $=$ guest \&checksum=1FF5543BAD8A658D0159E A68344EF7A5

OCDE. (2018). Doctors. Recuperado de https://data.oecd.org/healthres/doctors.htm

OCDE. (2018). Hospital beds. Recuperado de https://data.oecd.org/healtheqt/hospitalbeds.htm

OCDE. (2018). Nurses. Recuperado de https://data.oecd.org/healthres/nurses.htm\#indic ator-chart

OCDE. (2019). Health Statistics 2019. Recuperado de https://stats.oecd.org/Index.aspx ?DataSetCode= SHA

OMS. (1948). Preguntas Frecuentes. ¿Cómo define la OMS la salud? Recuperado de https://www.who.int/es/about/who-weare/frequently-asked-questions

OMS. (2005). ¿Qué es un sistema de salud? Recuperado https://www.who.int/features/qa/28/es/
OMS. (2016). Resumen del 2016:repaso de la salud mundial de la OMS. Recuperado de https://www.who.int/es/news-room/featurestories/detail/2016-year-in-review-key-healthissues

OPS. (2015). Calidad en el Sistema de Salud para el Acceso y la Cobertura Universal en Salud. Recuperado de https://www.paho.org/mex/index.php?option=c om_content\&view=article\&id=1023:calidaden-el-sistema-de-salud-para-el-acceso-y-lacobertura-universal-en-salud\&Itemid $=499$

Salinas, G., Carrillo, M.F., Pérez, M.U. y García, C. (2019). Gasto de bolsillo en salud durante el último año de vida de adultos mayores mexicanos: análisis del Enasem. Salud Pública de México, 61(4). pp. 510-511. Recuperado de http://www.saludpublica.mx/index.php/spm/arti cle/view/10146

Secretaría de Salud. (2015). La calidad de la atención a la salud en México a través de sus instituciones. Recuperado de http://www.calidad.salud.gob.mx/site/editorial/ dgr-editorial_02.html

Secretaría de Salud. (2016). Encuesta Nacional de Salud y Nutrición de Medio Camino 2016 (ENSANUT 2016) Informe Final de Resultados. Recuperado de http://transparencia.insp.mx/2017/auditoriasinsp/12701_Resultados_Encuesta_ENSANUT_ MC2016.pdf

Secretaría de Salud. (2019). Gasto en Salud en el Sistema Nacional de Salud. Recuperado de http://www.dgis.salud.gob.mx/contenidos/sinais /gastoensalud_gobmx.html

Senado de la República. (2018). Recursos destinados al Sector Salud en el Presupuesto de Egresos de la Federación 2018. Recuperado de http://bibliodigitalibd.senado.gob.mx/bitstream/ handle/123456789/3832/1\%20Publicaci\%C3\% B3n\%20Recursos\%20destinados\%20al\%20sect or\%20salud\%20PEF\%202018.pdf?sequence $=1$ \&isAllowed $=\mathrm{y}$

Vera, S. A. (2009). Bloque 2: Los Gastos Públicos. Recuperado de http://www.ispn4santafe.edu.ar/Carreras/Programador/Trabajos/ Finanzas_1.pdf 